\title{
Effects of type of general anaesthesia on markers of inflammatory response in elderly
}

\section{Ploshchenko Yulita, Klygunenko Olena \\ SE "Dnipropetrovsk Medical Academy of the MH of Ukraine", Department of anesthesiology, intensive care and emergency medicine FPE, Dnipro, Ukraine.}

Background: General anaesthesia may influence the inflammatory responses during the perioperative period. Dysregulation of the inflammatory process may provoke postoperative complications. An additional serious problem for anesthetists is the elderly patients and concomitant cardiovascular diseases. The incidence of inflammation in elderly is 20 to $30 \%$ [Nearman H. et al., 2014]. In $40 \%$ of patients promotes postoperative multiple organ dysfunction.

The goal of our study The goal of our study was to examine and evaluate markers of Inflammatory Response in elderly with concomitant cardiovascular diseases after general anaesthesia such as intravenous versus volative one.

\section{Material and Methods}

The study was performed in the clinic of Department of Anesthesiology, IC and EM.

We examined 40 patients aged 60 to 82 years for abdominal surgery with total intravenous or volative anesthesia. There were representative of the gender, age, ASA, BMI, Euroscore.

\begin{tabular}{|c|c|c|}
\hline Number of patients & 20 & 20 \\
\hline Male / Female & $12 / 8$ & $11 / 9$ \\
\hline mean age & 72,5 & 73,62 \\
\hline ASA II / III & $10 / 10$ & $9 / 11$ \\
\hline Body mass index & $28,1 \pm 6,86$ & $29,2 \pm 5,74$ \\
\hline EuroScore & $>5 \%$ & $>5 \%$ \\
\hline
\end{tabular}

Preoperative patients with CVD managed in accordance with ESC Guidelines $(2009,2014)$ Guidelines for pre - operative cardiac risk assessment and perioperative cardiac management in non - cardiac surgery.

All patients received treatment of All patients received pharmacological treatment of hypertension and myocardial ischemic disease according to the current guidelines to include: $\beta$ - blockers, nitrate, angiotensin converting enzyme inhibitors /BRA, part of the patients - with the use of calcium channel blockers and diuretics.

TNF, IL-6, IL-10 in EDTA-plasma determined by enzyme immunoassay (set Biomedica) (1) preoperative, (2) 1-st day, (3) 5-s day. Data are presented as $M \pm m$, statistically significant value of $p<0,05$.).

\section{Results and Discussion}

Preoperatively in both groops we did not observe any differences: the level of proinflammatory IL 6 exceeded the norm near 62-64,\%, TNF $\alpha$ - on 60-61\%, the level of antiinflammatory IL 10 exceeded the norm by $58-60 \%(p<0,01)$ in both groups without statistical differences. These changes were characteristic of elderly patients, which we observed in our previous works.

After anesthesia, the level of IL6 in the group with intravenous anesthesia was significantly higher than in patients with the group of inhaled anesthetics and increased near to $2651 \%(p=0.002)$. TNF alpha also increased significantly in the group with intravenous anesthesia from 1 day after surgery and slightly decreased by 5 days. The concentration of anti-inflammatory cytokine 10 , on the contrary, increased in the group of patients who received inhalation anesthesia.

The predominance of the proinflammatory response with the use of intravenous anesthesia was confirmed by high values of the IL6 / IL 10 coefficient.

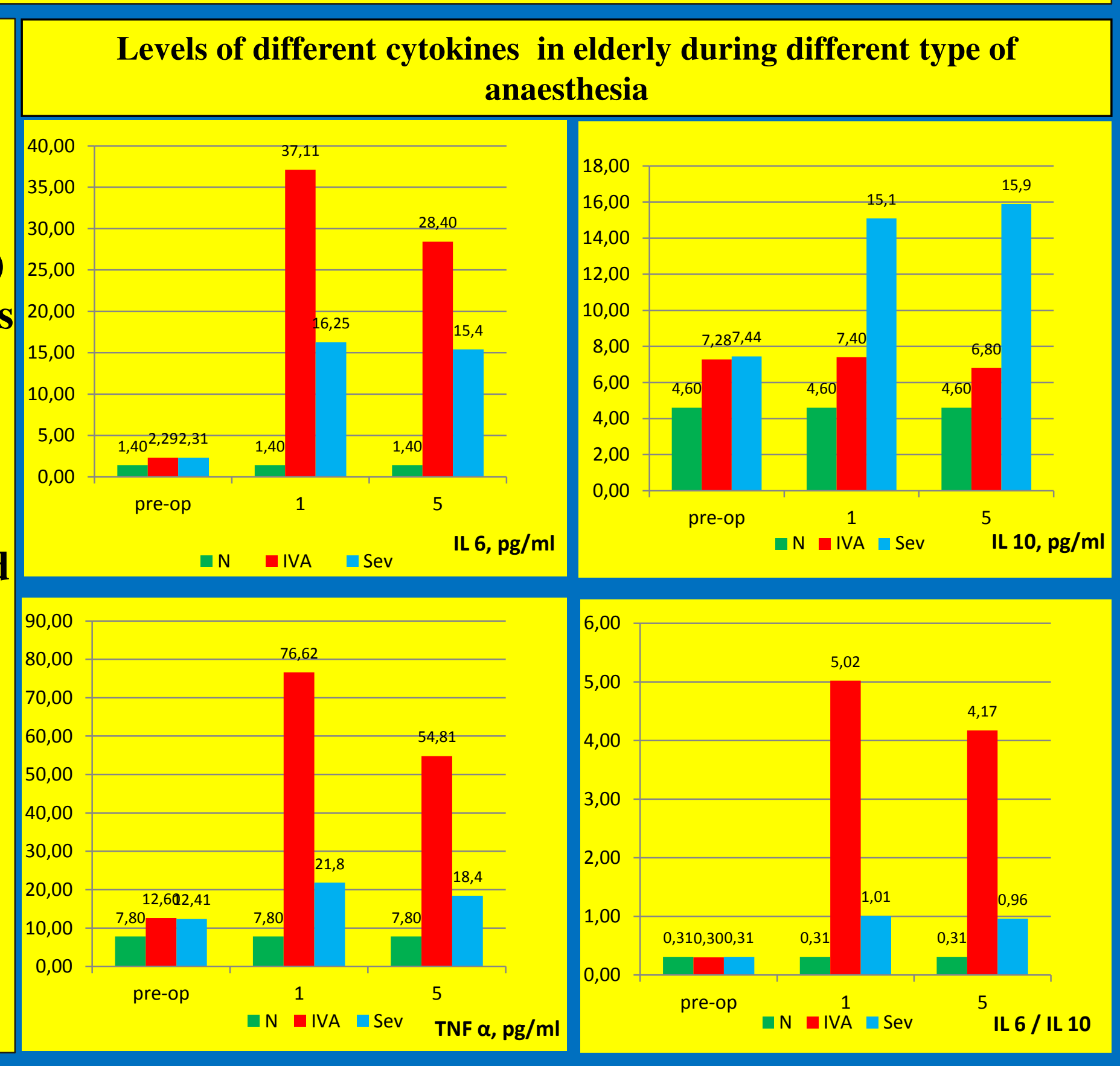

\section{Conclusion}

1. Elderly patients preoperative showed a non-specific activation of the inflammatory response that manifested as high in pro-inflammatory and anti-inflammatory cytokines.

2. After anesthesia in elderly we detected the inflammatory activation, which was confirmed by the increasing levels of cytokines and value index IL 6/IL 10.

3. After total intravenous anesthesia in elderly we detected the more intensive inflammatory activation than usage of inhalation one. 